\title{
Medición de óxido nítrico nasal: recomendaciones y procedimiento
}

\section{Measurement of nasal nitric oxide: recommendations and procedure}

\author{
Carlos Guzmán-Valderrábano,, * Wilmer Madrid-Mejía,* Prysila Troncoso-Huitrón,,\$ \\ Mónica Silva-Cerón,* Silvia Cid-Juárez,* Víctor Hernández-Morales, ${ }^{*}$ \\ Luis Torre-Bouscoulet, ${ }^{\S}$ Lissa De la Vega-Morales, ${ }^{\ddagger}$ Laura Gochicoa-Rangel*, \\ *Instituto Nacional de Enfermedades Respiratorias Ismael Cosío Villegas, Ciudad de México, México; ${ }^{\ddagger}$ PediaHero, Ciudad de
México; ${ }^{\S}$ Instituto de Desarrollo e Innovación en Fisiología Respiratoria S de RL, Ciudad de México.
}

RESUMEN. La medición del óxido nítrico nasal es una herramienta de gran utilidad para el diagnóstico de discinesia ciliar primaria. En comparación con sujetos sanos o con otras enfermedades respiratorias, los pacientes con discinesia ciliar primaria presentan bajas concentraciones de óxido nítrico nasal. La medición de óxido nítrico nasal es sencilla y requiere cooperación mínima de quien la realiza. El propósito de este documento es describir el procedimiento de medición de óxido nítrico nasal, así como revisar aspectos técnicos para la calibración y preparación del equipo.

Palabras clave: Óxido nítrico, óxido nítrico nasal, discinesia ciliar primaria.

\section{INTRODUCCIÓN}

El óxido nítrico $(\mathrm{NO})$ se forma cuando la L-arginina se oxida a L-citrulina a través de la acción enzimática de la óxido nítrico sintasa.' En el epitelio respiratorio, el NO lleva a cabo una función broncodilatadora, antimicrobiana e inmunomoduladora ${ }^{2-4}$ y se encuentra presente en mastocitos, neutrófilos, células cebadas y macrófagos del epitelio respiratorio. El NO difunde en forma gaseosa hacia la luz de la vía aérea, ${ }^{5-8}$ lo que facilita su medición que puede ser de forma indirecta por sus derivados biológicos, o directamente por quimioluminiscencia. ${ }^{2}$

Dentro del epitelio respiratorio, la mayor parte de la producción de NO se lleva a cabo en los senos paranasales, por lo que en la nariz las concentraciones de este gas son de $10 \mathrm{a}$ 100 veces mayores que las concentraciones de NO exhalado

Correspondencia:

Dra. Laura Graciela Gochicoa-Rangel

Instituto Nacional de Enfermedades Respiratorias Ismael Cosío

Villegas, Ciudad de México, México.

Correo electrónico: drgochis@gmail.com

Trabajo recibido: 30-X-2019; aceptado: 12-XI-2019
ABSTRACT. The measurement of nasal nitric oxide ( $\mathrm{nNO}$ ) has been a very useful tool for the diagnosis of primary ciliary dyskinesia (PCD). Compared with healthy subjects or affected by others respiratory diseases, $\mathrm{PCD}$ patients show low concentrations of $\mathrm{nNO}$. $\mathrm{nNO}$ is a simple test that requires minimal cooperation from the subject. The aim of this document is to review technical aspects for calibration, preparation and measurement of $\mathrm{nNO}$.

Keywords: Nitric oxide, nasal nitric oxide, primary ciliary dyskinesia.

de la vía respiratoria inferior. ${ }^{9}$ Existen diversas enfermedades que pueden estar asociadas con aumento o disminución de la concentración de $\mathrm{NO}$ en el epitelio respiratorio; por ejemplo, la inflamación del tracto respiratorio en sujetos con asma puede causar aumento de la concentración del NO exhalado por boca y nariz; mientras que en la inflamación debida a otras causas, como en el caso de las bronquiectasias idiopáticas, puede existir un aumento de $\mathrm{NO}$ exhalado por boca, pero estar disminuido o normal por vía nasal.10,11

En algunos casos, los pacientes con sinusitis pueden presentar incremento o disminución de óxido nítrico nasal (nNO) dependiendo de los mecanismos inflamatorios subyacentes..$^{12,13}$ En pacientes con discinesia ciliar primaria (DCP), las concentraciones de NO exhalado y nasal son considerablemente más bajas en comparación con sujetos sanos y pacientes con otras enfermedades respiratorias. Actualmente, la medición de nNO se considera como parte de las pruebas diagnósticas para esta enfermedad. 14,15

\section{Discinesia ciliar primaria}

La DCP es una enfermedad que afecta aproximadamente a 1/15,000 a 1/30,000 nacidos vivos. La mayoría de los 
Neumol Cir Torax. 2020; 79 (1): 53-58

casos son por herencia autosómica recesiva que se caracteriza por un espectro de defectos en la ultraestructura o la función ciliar. ${ }^{16,17}$ El deterioro de la función ciliar impide la depuración mucociliar, lo que predispone en el $75 \%$ de la población enferma a la presentación de los síntomas clásicos de la DCP que incluyen congestión nasal crónica, sinusitis crónica, otitis media recurrente, tos productiva, neumonías o bronquitis recurrentes, así como dificultad respiratoria en el período neonatal. El 50\% de los individuos afectados tiene situs inversus totalis; y en algunos casos, se han reportado situs ambiguos o heterotaxia.,18

La microscopía electrónica de transmisión (ME) de células epiteliales ciliadas, ha sido considerada el estándar de oro en el diagnóstico de esta enfermedad. Mediante la ME se evalúan las anormalidades ultraestructurales de los cilios respiratorios, los brazos de dineína internos y externos, el haz radial o el aparato central. ${ }^{1}$ El procesamiento e interpretación de las muestras ciliares mediante ME requiere una amplia capacitación y experiencia por lo que se limita a pocos centros de atención de tercer nivel y es poco accesible en algunos países. De igual manera, estudios recientes han demostrado que los resultados de ultraestructura ciliar pueden ser normales en algunos casos de DCP confirmados por pruebas genéticas. ${ }^{19}$ Por lo tanto, se ha puesto mayor énfasis en otras pruebas para el abordaje diagnóstico de esta enfermedad como la medición nNO. Esta es una prueba accesible, mínimamente invasiva y con alta sensibilidad y especificidad para el diagnóstico de DCP.

\section{Medición de nNO para diagnóstico de discinesia ciliar primaria}

El estándar de oro para la medición de $\mathrm{NO}$ es el método de quimioluminiscencia. Se basa en la medición de la intensidad de radiación fluorescente emitida después de la oxidación química del NO por el ozono. El producto de esta reacción es el dióxido de nitrógeno (NO2), se emite un fotón por cada reacción. La cantidad de fotones es proporcional a la concentración de $\mathrm{NO}$ en el tejido o gas que se esté analizando. ${ }^{4}$

La DCP se caracteriza por concentraciones bajas de nNO. En niños mayores de cinco años y en adultos, un punto de corte $<77 \mathrm{~nL} /$ min otorga sensibilidad de $97.5 \%$ y especificidad de $96.4 \%,{ }^{20,21}$ mientras que los controles sanos suelen presentar niveles superiores a $250 \mathrm{~nL} / \mathrm{min} .^{22,23}$

La guía de práctica clínica para diagnóstico de DCP de la Sociedad Americana de Tórax (ATS), recomienda que en sujetos mayores de cinco años de edad con fenotipo clínico consistente con DCP y habiendo descartado fibrosis quística, una concentración de $\mathrm{nNO}<77 \mathrm{~nL} / \mathrm{min}$, medido en dos ocasiones en días distintos, es suficiente para sostener el diagnóstico de DCP. En esas circunstancias, la ME o las pruebas genéticas contribuyen a precisar el pronóstico y mejorar la comprensión de la enfermedad. ${ }^{21}$

\section{PROCEDIMIENTO PARA LA MEDICIÓN DE NNO MEDIANTE EL ANALIZADOR DE ÓXIDO NÍTRICO SIEVERS ${ }^{\circledR}$ NOA 2801}

La medición en línea del nNO es una prueba rápida y no invasiva para la detección de DCP. El procedimiento completo toma de 10 a 20 minutos y los resultados están disponibles inmediatamente. Este estudio se realiza con analizadores de óxido nítrico (AON) por el método de quimioluminiscencia y la maniobra debe realizarse bajo una metodología estandarizada.

\section{Calibración del equipo}

Existen diversos factores como la humedad, el ambiente, el voltaje de línea y la cámara de reacción que pueden afectar la calibración de los equipos para medición de ON. Se debe llevar a cabo una calibración diariamente de estos equipos que consiste en dos partes; la primera, es con un gas con una concentración de $\mathrm{ON}<1$ parte por billón (ppb) (Gas Cero); y la segunda con un gas con concentración conocida de ON.

- Gas cero: para la calibración del Gas Cero se utiliza un filtro que contiene permanganato de potasio y carbón activado, conectado a la línea de muestreo de NO. Se activa entonces el equipo para que se genere un flujo de aspiración de $200 \mathrm{~mL} / \mathrm{min}$. Al pasar el aire a través
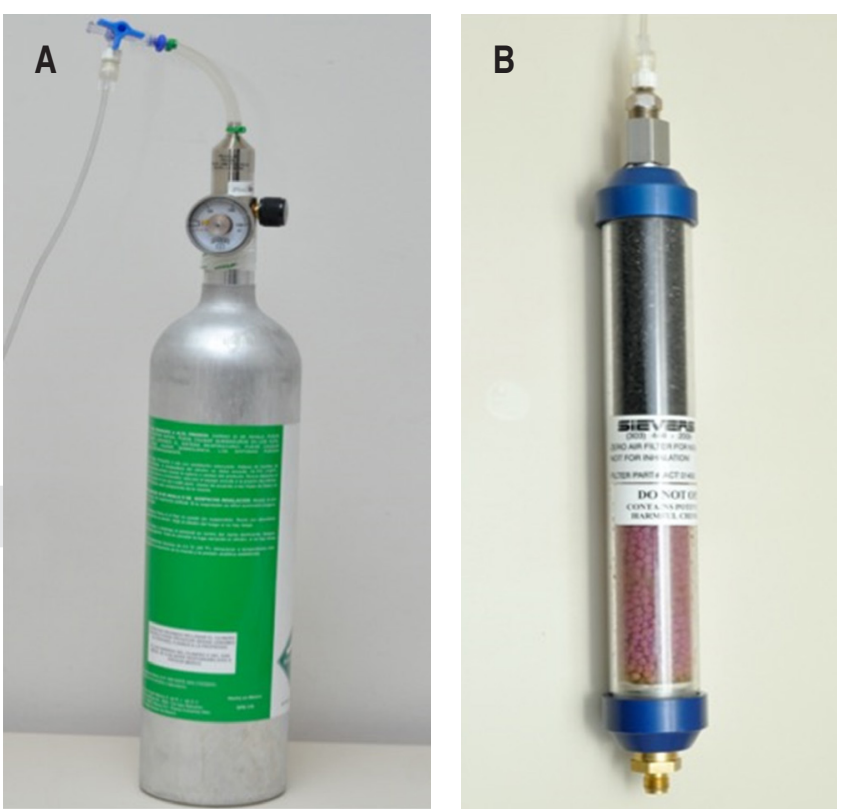

Figura 1: Material para calibración. A) tanque con gas con una concentración de NO de 45 ppm; B) filtro de permanganato de potasio y carbón activado para calibración con gas cero. 
de $\sim 90 \mathrm{mV}$. Por lo tanto, el AON calcula la respuesta para el rango ppb basándose en la nueva calibración en $\mathrm{ppm}^{24}$ (Figura 1).

\section{Recomendaciones antes de la prueba}

Se debe realizar el estudio cuando el sujeto se encuentra clínicamente estable. Se recomienda:

- No haber presentado infección respiratoria en las últimas dos semanas.

- No haberse realizado instrumentación nasal en las últimas dos semanas cirugía nasal, biopsia de cornetes y nasofibroscopía).

- Evitar tabaquismo, consumo de alimentos ricos en nitratos (lechuga) y bebidas con cafeína dos horas antes de la prueba.

- No haber ingerido alcohol 12 horas antes de la prueba.

- No haber hecho ejercicio 30 minutos antes de la prueba.

Figura 2: Analizador de óxido nítrico Sievers ${ }^{\circledR}$ NOA $280 \mathrm{i}$.

del filtro, éste produce oxidación a $\mathrm{NO}_{2}$, el cual es absorbido por el carbón activado dejando pasar a la línea de muestreo aire sin NO; por lo tanto, el resultado de la maniobra es cero. Se deben realizar mínimo dos calibraciones para replicar resultados estables y después el equipo hace un ajuste de compensaciones de ppb y partes por millón (ppm).

- Calibración de gas a una concentración conocida: se utiliza un gas con una concentración de NO entre 10-100 ppm, usualmente $45 \mathrm{ppm}$. Se conecta a la línea de muestreo del AON mediante una pieza «T» proporcionando un flujo de más de $200 \mathrm{~mL} / \mathrm{min}$ (casi siempre $300 \mathrm{~mL} / \mathrm{min}$ dada por el regulador). El equipo Sievers ${ }^{\circledR}$ NOA 280i está ajustado de fábrica para tener una respuesta de $2 \mathrm{mV} / \mathrm{ppm}$ por lo que para un gas de calibración de 45 ppm, la señal de voltaje final debe ser

\section{Equipo y materiales}

- Analizador de NO por quimioluminiscencia (Sievers ${ }^{\circledR}$ NOA 280i ${ }^{\circledR}$ ) (Figura 2).

- Catéter de plástico con oliva o esponja nasal suave.

- Resistor desechable para generar una resistencia superior a $5 \mathrm{cmH}_{2} \mathrm{O}$ y garantizar un cierre del paladar blando (cilindro de cartón con una abertura de $1 \mathrm{~mm}$ en el extremo distal o juguete de fiesta para soplar «espanta suegras») (Figura 3).

\section{Técnica}

- Estando el sujeto sentado se coloca el catéter de plástico con esponja en una narina, lo suficientemente profundo para garantizar un sellado hermético (Figura 4).
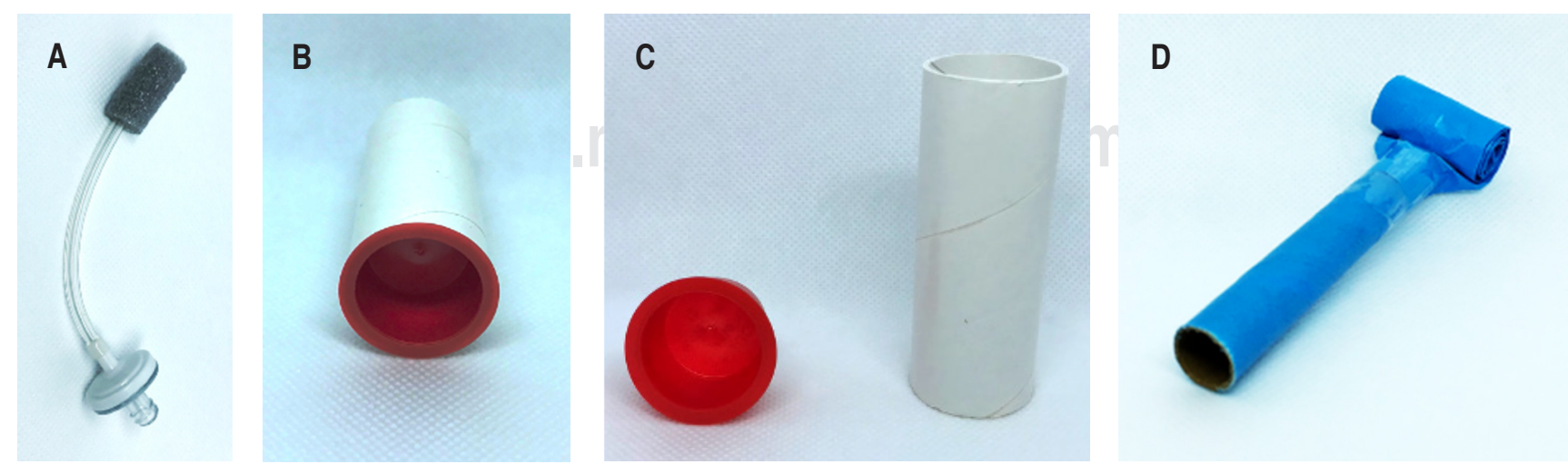

Figura 3: Materiales consumibles para realización de la prueba: A) catéter para aspiración con esponja nasal; B y C) resistor de plástico con orificio de $1 \mathrm{~mm}$ y boquilla de cartón desechable; D) juguete de fiesta para soplar «espanta suegras». 
- Se solicita al sujeto que inspire profundo y después que coloque el resistor desechable en su boca y que exhale lentamente durante aproximadamente 20-40 segundos.

- Mientras el sujeto exhala por la boca, el gas nasal es aspirado por el equipo a través del catéter insertado en la fosa nasal.

\section{Criterios de aceptabilidad y repetibilidad}

- Exhalación de más de 10 segundos.

- Representación gráfica en pantalla de una meseta de la señal de NO constante durante 3 a 10 segundos (Figura 5).

- Después de dos maniobras aceptables se evalúa la repetibilidad. Consiste en que la diferencia entre las dos
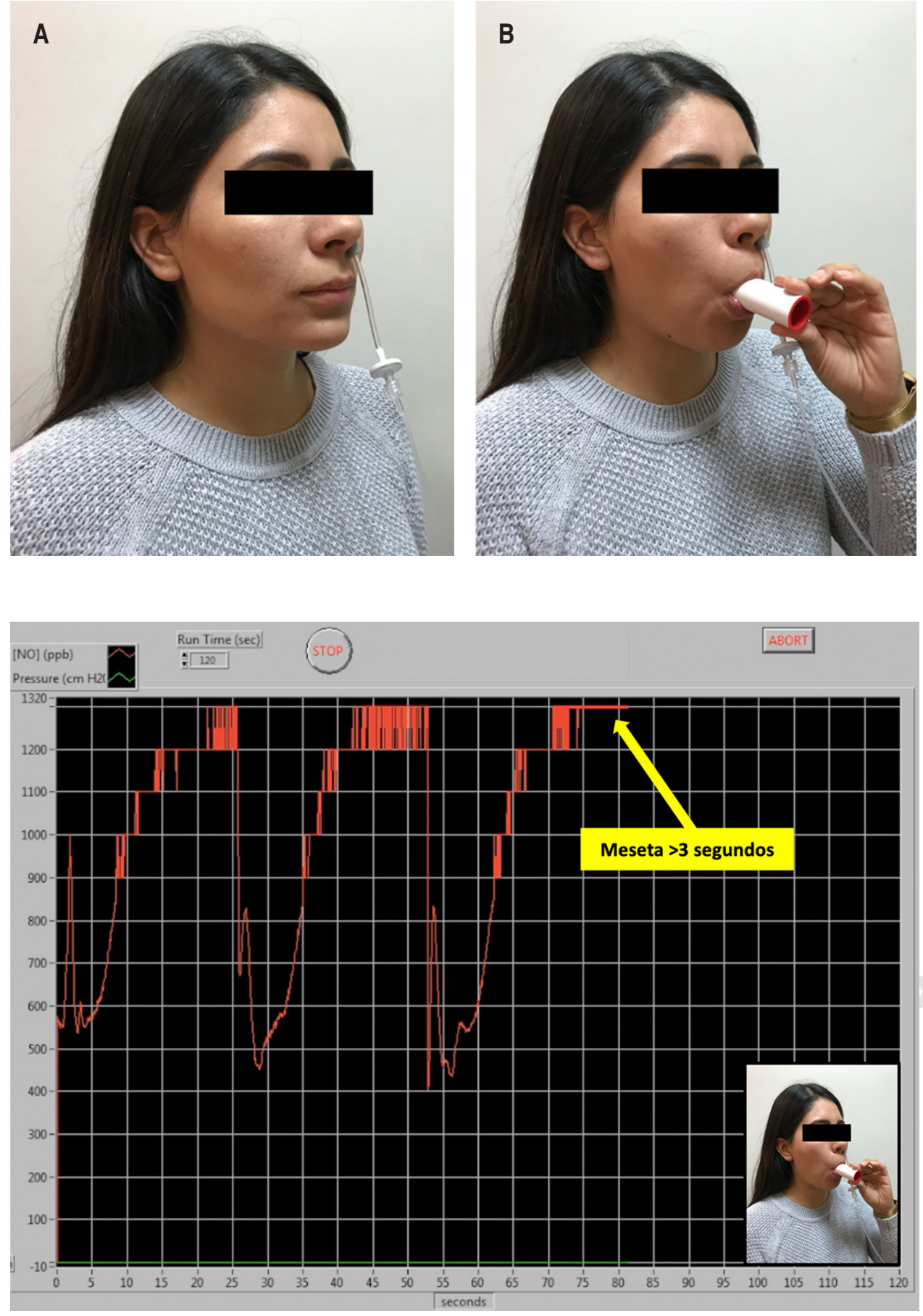

\section{Figura 4:}

Preparación del individuo para realización de la maniobra de $\mathrm{nNO}$. A) Colocación de catéter con esponja nasal en una fosa nasal dejando libre la otra fosa nasal; B) exhalación sostenida a través de la boquilla de cartón desechable con el resistor.

\section{Figura 5:}

Gráfico de la medición de nNO durante la exhalación. Se observa la meseta de al menos tres segundos (flecha). 
Sievers Instruments Model 280/280I NOA

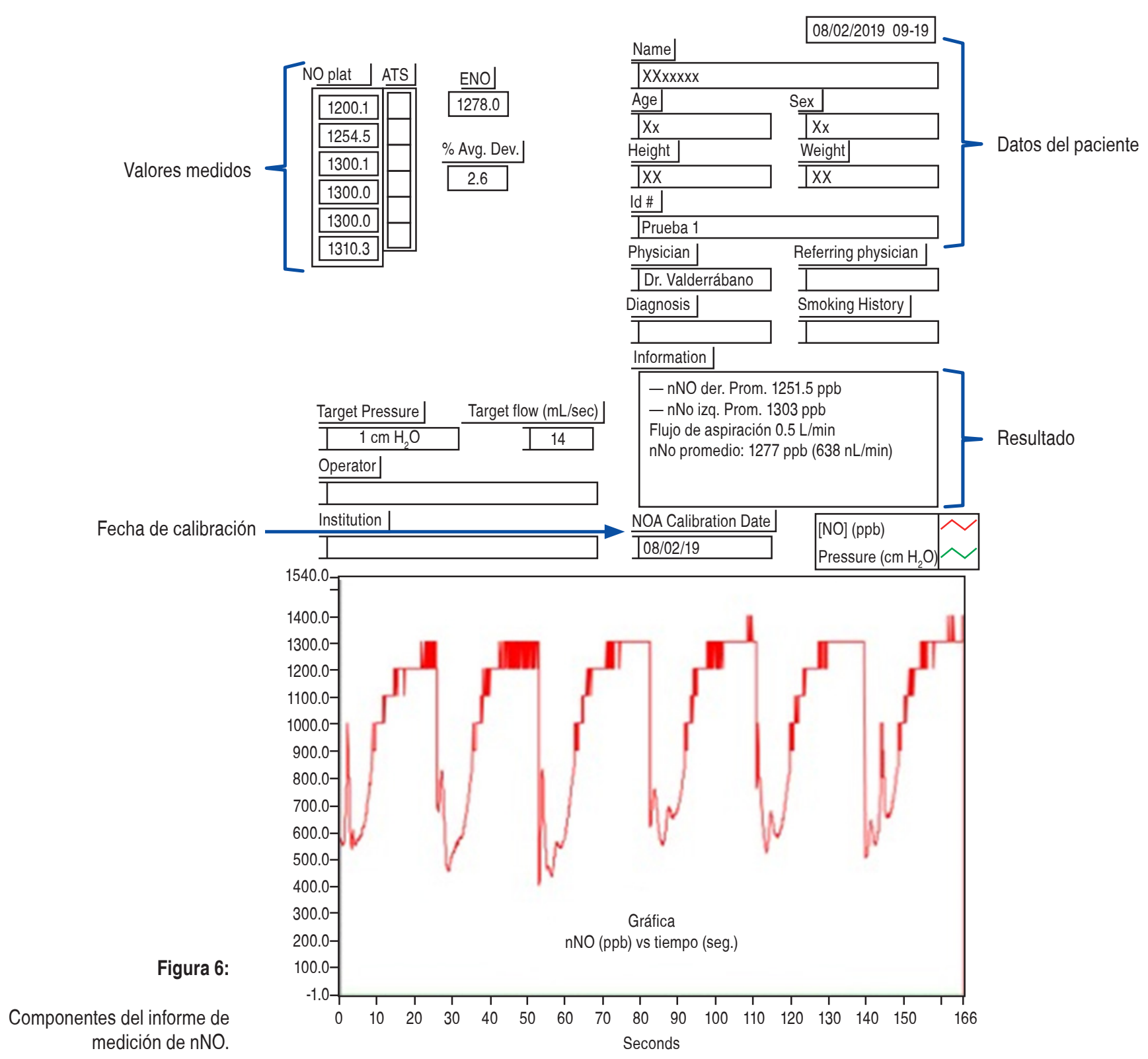

mediciones aceptables sea menor al 10\%. El mismo procedimiento se realiza en la otra narina.

\section{Informe de resultados}

El resultado del valor medido de cada fosa nasal se considera como la media de los valores medidos y se reporta como resultado final la media de las medias de los valores de $\mathrm{nNO}$ de ambas fosas nasales (derecha e izquierda). ${ }^{25}$ Debido a que los valores de $\mathrm{nNO}$ son reportados en $\mathrm{ppb}$ será necesario hacer la conversión a nL/min multiplicando la concentración de nNO en ppb por el flujo de muestreo del equipo de quimioluminiscencia que se esté utilizando $\left(0.5 \mathrm{~L} / \mathrm{min}\right.$ en el caso del equipo Sievers $\left.{ }^{\circledR} \mathrm{NOA} 280 \mathrm{i}\right)$ (Figura 6).

El reporte generado debe contener la información necesaria para poder identificar al sujeto a quien se le realizó el estudio, las características del equipo, fecha de última calibración y datos de la maniobra, que permitan evaluar la calidad del estudio realizado:

- Nombre completo del paciente.

- Parámetros antropométricos (edad, género, peso y estatura). 
Neumol Cir Torax. 2020; 79 (1): 53-58

- Fecha de última calibración.

- Valores individuales de cada una de las mediciones realizadas.

- Gráfica que muestre la concentración de ON (ppb) versus gráfico de tiempo (segundos).

- Media de los valores medidos de cada fosa nasal en $\mathrm{ppb}$ (nNO derecho, $\mathrm{nNO}$ izquierdo).

- Resultado final de $\mathrm{nNO}$ en ppb y en $\mathrm{nL} / \mathrm{min}$.

\section{CONCLUSIONES}

Un protocolo estandarizado para la medición de nNO contribuye de manera eficiente, accesible, sencilla y mínimamente invasiva a una evaluación precisa y diagnósticos certeros en los individuos con DCP.

\section{REFERENCIAS}

1. Davis SD, Eber E, Koumbourlis AC, American Thoracic Society, editors. Diagnostic tests in pediatric pulmonology: applications and interpretation. New York: Humana Press; 2015.p. 321.

2. Cantú-González G, Fernández-Figueroa F, Gochicoa-Rangel L, et al. Fracción exhalada de óxido nítrico. Recomendaciones clínicas y procedimiento. Neumol Cir Torax 2013;72(1):43-51.

3. Lane C, Knight D, Burgess S, et al. Epithelial inducible nitric oxide synthase activity is the major determinant of nitric oxide concentration in exhaled breath. Thorax 2004;59(9):757-760. https:// doi.org/10.1136/thx.2003.014894.

4. Ricciardolo FL. Multiple roles of nitric oxide in the airways. Thorax 2003;58(2):175-182. https://doi.org/10.1136/thorax.58.2.175.

5. de Diego Damiá A. Nitric oxide in asthma: what good does it do? Arch Bronconeumol 2010;46(4):157-159. https://doi.org/10.1016/j. arbres.2009.12.010.

6. Nathan C, Xie QW. Nitric oxide synthases: roles, tolls, and controls. Cell 1994;78(6):915-918. https://doi.org/10.1016/00928674(94)90266-6.

7. Ignarro LJ, Buga GM, Wood KS, Byrns RE, Chaudhuri G. Endothelium-derived relaxing factor produced and released from artery and vein is nitric oxide. Proc Natl Acad Sci USA 1987;84(24):9265-9269. https://doi.org/10.1073/pnas.84.24.9265.

8. Palmer RM, Ashton DS, Moncada S. Vascular endothelial cells synthesize nitric oxide from L-arginine. Nature 1988;333(6174):664666. https://doi.org/10.1038/333664a0.

9. Lundberg JO. Nitric oxide and the paranasal sinuses. Anat Rec 2008;291(11):1479-1484. https://doi.org/10.1002/ar.20782.

10. Narang I, Ersu R, Wilson NM, Bush A. Nitric oxide in chronic airway inflammation in children: diagnostic use and pathophysiological significance. Thorax 2002;57(7):586-589. https://doi.org/10.1136/ thorax.57.7.586.

11. Manna A, Montella S, Maniscalco M, Maglione M, Santamaria F. Clinical application of nasal nitric oxide measurement in pediatric airway diseases. Pediatr Pulmonol 2015;50(1):85-99. https://doi. org/10.1002/ppul.23094.

12. Arnal JF, Flores P, Rami J, et al. Nasal nitric oxide concentration in paranasal sinus inflammatory diseases. Eur Respir J 1999;13(2):307712. https://doi.org/10.1034/j.1399-3003.1999.13b15.x.
13. Bommarito L, Guida G, Heffler E, et al. Nasal nitric oxide concentration in suspected chronic rhinosinusitis. Ann Allergy Asthma Immunol 2008;101(4):358-362. https://doi.org/10.1016/ S1081-1206(10)60310-9

14. Balfour-Lynn IM, Laverty A, Dinwiddie R. Reduced upper airway nitric oxide in cystic fibrosis. Arch Dis Child 1996;75(4):319-322. https://doi.org/10.1136/adc.75.4.319.

15. Collins SA, Gove K, Walker W, Lucas JSA. Nasal nitric oxide screening for primary ciliary dyskinesia: systematic review and meta-analysis. Eur Respir J 2014;44(6):1589-1599. https://doi. org/10.1183/09031936.00088614.

16. Katsuhara K, Kawamoto S, Wakabayashi T, Belsky JL. Situs inversus totalis and Kartagener's syndrome in a Japanese population. Chest 1972;61(1):56-61. https://doi.org/10.1378/ chest.61.1.56.

17. Chilvers MA, Rutman A, O'Callaghan C. Ciliary beat pattern is associated with specific ultrastructural defects in primary ciliary dyskinesia. J Allergy Clin Immunol 2003;112(3):518-524. https:/l doi.org/10.1016/s0091-6749(03)01799-8.

18. Knowles MR, Daniels LA, Davis SD, Zariwala MA, Leigh MW. Primary ciliary dyskinesia. Recent advances in diagnostics, genetics, and characterization of clinical disease. Am J Respir Crit Care Med 2013;188(8):913-922. https://doi.org/10.1164/ rccm.201301-0059Cl.

19. Knowles MR, Leigh MW, Carson JL, et al.; Genetic Disorders of Mucociliary Clearance Consortium. Mutations of DNAH11 in primary ciliary dyskinesia with normal ciliary ultrastructure. Thorax 2012;67(5):433-441. https://doi.org/10.1136/ thoraxjnl-2011-200301.

20. Leigh MW, Hazucha MJ, Chawla KK, et al. Standardizing nasal nitric oxide measurement as a test for primary ciliary dyskinesia. Ann Am Thorac Soc 2013;10(6):574-581.https://doi.org/10.1513/ AnnalsATS.201305-1100C.

21. Shapiro AJ, Davis SD, Polineni D, et al.; American Thoracic Society Assembly on Pediatrics. Diagnosis of primary ciliary dyskinesia. An Official American Thoracic Society Clinical Practice Guideline. Am J Respir Crit Care Med 2018;197(12):e24-e39. https://doi.org/10.1164/ rccm.201805-0819ST.

22. Karadag B, James AJ, Gültekin E, Wilson NM, Bush A. Nasal and lower airway level of nitric oxide in children with primary ciliary dyskinesia. Eur Respir J 1999;13(6):1402-1405.

23. Lundberg JO, Weitzberg E, Nordvall SL, Kuylenstierna R, Lundberg JM, Alving K. Primarily nasal origin of exhaled nitric oxide and absence in Kartagener's syndrome. Eur Respir J 1994;7(8):1501-1504. https://doi.org/10.1183/09031936.94.0708 1501.

24. GE Analytical Instruments ${ }^{\circledR} 2006$. Sievers ${ }^{\circledR}$ NOA 280i. Operation and Maintenance Manual. Firmware version 3.00 and later.

25. ATS/ERS recommendations for standardized procedures for the online and offline measurement of exhaled lower respiratory nitric oxide and nasal nitric oxide, 2005. Am J Respir Crit Care Med 2005;171(8):912-930. https://doi.org/10.1164/rccm.200406710 ST.

Conflicto de intereses: Los autores declaran no tener conflicto de intereses. 